\title{
Utilización de las TIC en el proceso de enseñanza - aprendizaje, una aproximación desde la comunicación
}

\section{Use of ICT in the teaching - learning process, an approach from communication}

Bismarck Alberto Arana Mite

Universidad Politécnica Salesiana, Ecuador

María José Segarra Sanz

Universidad Politécnica Salesiana, Ecuador

Autor para correspondencia: barana@ups.edu.ec, msegarra@est.ups.edu.ec

Fecha de recepción: 05 de Agosto de 2017 - Fecha de aceptación: 10 de Agosto de 2017

\section{Resumen}

El presente trabajo analiza la función e importancia que cumplen las TIC en el proceso de enseñanza - aprendizaje en la Unidad Educativa de la Providencia de la ciudad de Guayaquil. El uso de las herramientas virtuales dentro de la institución propone un primer acercamiento a la realidad educativa que servirá de base para la implementación de nuevas técnicas que parten del uso de las TIC. Se desarrolló una investigación con enfoque mixto mediante entrevistas y encuestas realizadas a docentes de la institución, lo que permitió saber cómo los estudiantes acceden y se involucran con la tecnología reemplazando la metodología tradicional de enseñanza.

Palabras Clave: herramientas tecnológicas; técnicas educativas; educación básica; comunicación

\begin{abstract}
This paper will analyze the role and importance of ICT in the teaching-learning process in the Educational Unit of the Providence of the city of Guayaquil. The use of the virtual tools within the institution proposes a first approach to the educational reality which will serve as the basis for the development of new imaging techniques that are based on the use of ICTS. A joint approach with research through surveys and interviews to teachers of the institution, which will allow you to know how students access and are involved with the technology replacing the traditional methodology of teaching.
\end{abstract}

Key words: technological tools; educational techniques; basic education; communication 


\section{Introducción}

Con las aportaciones de la tecnología y los conocimientos expuestos por el docente en el manejo de las TIC, se destaca la transformación del proceso enseñanza-aprendizaje para el correcto manejo de la información dentro de una institución educativa.

Las TIC aportan a la construcción de nuevos avances tecnológicos facilitando la participación e interacción en las prácticas pedagógicas. A su vez, favorecen el desempeño de los educadores mediante la utilización de aplicaciones o softwares que facilitan el aprendizaje de los estudiantes.

La Unidad Educativa de la Providencia, pertenece a la Congregación de Las Hijas de la Caridad como centro integral educativo ubicado en la ciudad de Guayaquil-Ecuador, específicamente en las calles Eloy Alfaro entre Gómez Rendón y Brasil. Esta institución se centra en los principios éticos y morales de la fe cristiana contando con instalaciones de óptima calidad para su alumnado. Los niveles de educación con los que cuenta la Unidad Educativa de la Providencia son Inicial, Básica y Bachillerato.

En los sectores vulnerables donde aún no ha llegado la tecnología, los docentes envían a sus alumnos a investigar en libros (método tradicional) por la falta de equipos y herramientas que les permita complementar su aprendizaje logrando una comunicación basada en los nuevos criterios de educación didáctica. Por tanto, se realiza el planteamiento de la siguiente interrogante ¿Cómo influye el uso de las Tic en el proceso de enseñanza- aprendizaje en la Unidad Educativa de la Providencia?

En todo momento se suscitan cambios que aportan a la educación en base a procesos didácticos educativos actuales, porque de cierta forma se complementan con los avances tecnológicos contribuyendo al desarrollo de la comunicación y optimizando la fluidez de la información en el ámbito educativo.

La importancia de las TIC en la formación educativa se ha convertido en un desafío más de los docentes, debido a que no todos son nativos digitales, sino que basan sus conocimientos en la metodología tradicional de enseñanza. Actualmente los docentes implementan en su plan de trabajo escolar el uso de softwares educativos incluyendo herramientas básicas como Word, Excel, Power Point.

\section{Las TIC en la educación}

La sociedad postindustrial ha sido catalogada como la sociedad de la nueva educación debido al uso preponderante de estrategias tecnológicas implementadas en la educación en diferentes ámbitos, por lo tanto, el movimiento innovador de la educación moderna en contraposición a la escuela tradicional se desarrolló a finales del siglo XIX en Europa propagándose en otros países del mundo, pero se erradicó con mayor fuerza en los Estados Unidos. Se confronta un gran cambio de época donde no solo se obtiene una Sociedad de la Información, sino una sociedad de la educación de manera didáctica. (Rugeles, Mora, \& Metaute, 2015) 
Cuando se habla de las Tecnologías de la Información y Comunicación (TIC) se piensa en los avances tecnológicos que permiten la obtención de nuevos conocimientos en base a los cambios presentados a lo largo del tiempo, esto significa que existe un mejor acceso a las herramientas digitales que permiten la construcción de conocimientos de forma progresiva.

El papel que desempeñan las TIC dentro de la educación básica es la de innovar los procesos de enseñanzas mediante el cual el docente impartirá sus clases haciéndolas más dinámicas y entretenidas. Para que esto suceda se debe complementar con el acceso a las nuevas tecnologías que permitan el fortalecimiento y desarrollo del ámbito educativo y de quienes son participes en él. (Gómez \& Macedo, 2012).

En las instituciones educativas existen nexos entre la comunicación y la educación por las innovaciones de las tecnologías, enfatizando que la información es un instrumento para el conocimiento de la sociedad que genera cambios y transcendencia en la educación (Carmona, Carmona, Armenta, Gastelú, \& García, 2016).

El uso del internet es un aporte dentro de las instituciones educativas que permite el desarrollo y evolución abarcando el manejo de diversas plataformas virtuales en las que docentes y alumnos participen paulatinamente implementando las nuevas tecnologías en el proceso de enseñanza de los jóvenes hoy en día.

Las TIC aportan la construcción de nuevos avances tecnológicos facilitando la participación e interacción en las prácticas pedagógicas. A su vez, favorecen el desempeño de los educadores mediante la utilización de aplicaciones o softwares que facilitan el aprendizaje de los estudiantes.

\section{Conceptualización y aspectos de la Educomunicación}

Según el libro “Ámbitos y alcances de la competencia comunicativa en educación“ Kaplún expresó: "Educar es, en esencia, un proceso de comunicación. Esta es una máxima que supone la vinculación del docente, responsable de la conducción, orientación y guía de la secuencia y orden de complejidad del aprendizaje con los educandos" (Cortés, Rigoberto, Uribe, \& Ibarra, 2013)

La educación va a la par con la comunicación, porque sin duda los procesos comunicativos se han desarrollado a medida que la tecnología ha brindado sus aportes a la sociedad actualmente y aún más al ámbito educativo.

La Educomunicación busca transformar los modelos tradicionales de estudio constituyendo modelos comunicativos que involucren la tecnología moderna, es decir, la fusión teórica - práctica para alcanzar que los centros educativos transformen la realidad monótona de aprendizaje por un entorno constituido por prácticas abiertas, en las que los estudiantes pongan en consideración la capacidad de interacción, comprensión y habilidades con la finalidad de innovar e ir en busca de nuevas tendencias pedagógicas adaptadas a un modelo de enseñanza que aporten a la formación integral de niños, adolescentes y jóvenes. 
El manejo de herramientas tecnológicas es una de tantas experiencias informativas que adopta el ser humano en una sociedad hipermedial que se ha convertido en algo significativo en vista de que nadie se educa solo, todos alguna vez necesitamos ayuda en complemento con la tecnología. Sin embargo, existen métodos en los cuales se pueda reflejar a la educación como base transformadora de conocimientos.

Las nuevas tecnologías de la información y la comunicación aplicadas a la educación, seguirán siendo un elemento esencial debido a que siempre se busca proyectar y conseguir efectos formativos en alumnos de niveles de educación básica obteniendo un mejor desempeño educativo, cabe destacar que las diferentes herramientas o plataformas virtuales también juegan un papel importante en todo tipo de método educativo por la influencia y contribuciones que estos aporten a los niños y jóvenes dentro y fuera de una institución escolar, adaptándose a los cambios positivos que entes transformadores (Ángel, 2013).

A la vez educarse es involucrarse y participar en un proceso de múltiples interacciones educomunicacionales, donde, generalmente el individuo que este en etapa de educación ha tenido que pasar por situaciones que ponen en riesgo su estabilidad educativa, sin embargo, es de suma importancia tratar de esclarecer cuáles son los aspectos que de alguna manera inciden en el avance y desarrollo del actual crecimiento didáctico pedagógico (Of, 2012).

\section{Comunicación y educación tecnológica en la educación básica}

El termino comunicación se ha enlazado a la educación para complementar los avances que trae consigo la tecnología, cuando el docente comunica o enseña algo dentro de las aulas de clase el alumno recibe la información logrando que se dé el entendimiento por parte del alumno. Destacando que la comunicación abarca el manejo o acceso a las herramientas tecnológicas en la educación actual (Vidal Puga, 2006).

La comunicación está presente en todo momento, para dar un ejemplo claro se toma en cuenta la unidad educativa de la Providencia donde se reflejará el manejo de herramientas tecnológicas como las computadoras, infocus o plataformas virtuales que permitan la interacción entre los docentes y alumnos. Tanto así que al alumno se le ha dado el nombre de estudiante virtual porque en la actualidad son capaces de seleccionar su propia información generando contenidos que les servirán como objeto de estudio junto a los refuerzos del profesor (Rúgeles et al., 2015).

La autodisciplina es lo que permite al alumno la motivación de seguir incursionando en el mundo tecnológico actual, y para que pueda lograr sus metas debe existir de alguna forma el autoaprendizaje facilitándole al estudiante virtual el desarrollo intelectual mediante manejo de información que aporte a su conocimiento, implementando la responsabilidad de tiempo y acceso a plataformas hipermediales. (Rúgeles et al., 2015)

\section{Metodología}


La investigación se desarrolló mediante una metodología mixta, complementada a través de encuestas de opinión y una entrevista, además se utilizó el programa estadístico SPSS para la interpretación de datos de la información proporcionada por los encuestados.

Del universo de 61 docentes de la institución educativa, la muestra seleccionada fue de 25 docentes que son quienes laboran en el área de educación básica.

\section{Resultados}

El disponer de herramientas tecnológicas actualmente es muy importante para el desarrollo del proceso enseñanza aprendizaje en los estudiantes de la institución educativa.

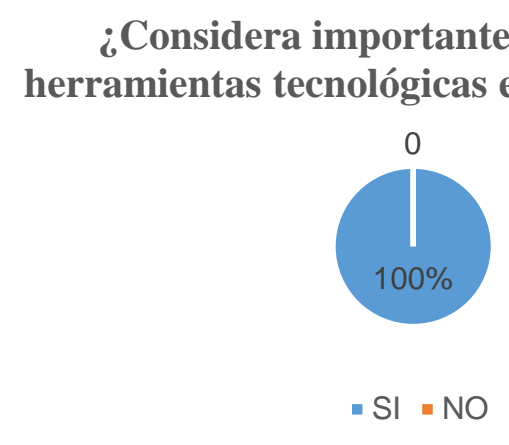

Gráfico 1. Las TIC en la actualidad Fuente: Elaboración propia

\section{Tabla 1.}

Considera importante disponer de herramientas tecnológicas en la actualidad?

\begin{tabular}{ccc}
\hline SI & NO & TOTAL \\
25 & 0 & 25 \\
$100 \%$ & $0 \%$ & $100 \%$ \\
\hline
\end{tabular}

Fuente: Elaboración propia

Todos los docentes encuestados coincidieron que la tecnología va evolucionando y la necesidad de utilizarla es cada vez más frecuente. 


\section{¿Considera que la TIC son importantes para la educación de hoy?}

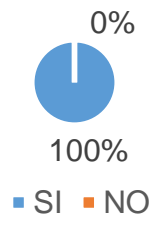

Gráfico 2. Importancia de las TIC

Fuente: Elaboración propia

Tabla 2.

¿Considera que las TIC son importantes para la educación de hoy?

\begin{tabular}{ccc}
\hline SI & NO & TOTAL \\
25 & 0 & 25 \\
$100 \%$ & $0 \%$ & $100 \%$ \\
\hline
\end{tabular}

Fuente: Elaboración propia

Los 25 docentes encuestados contestaron que las TIC sí son importantes para la educación, tal como se evidencia en la tabla, el 100\% se manifestó a favor del uso de las TIC.

\section{Nivel de tecnología en la institución educativa}

Nivel Alto: 10 docentes

Nivel Medio: 14 docentes

Nivel Bajo: 1 docente

¿Cuál es el nivel de tecnología en la

institución educativa donde labora?

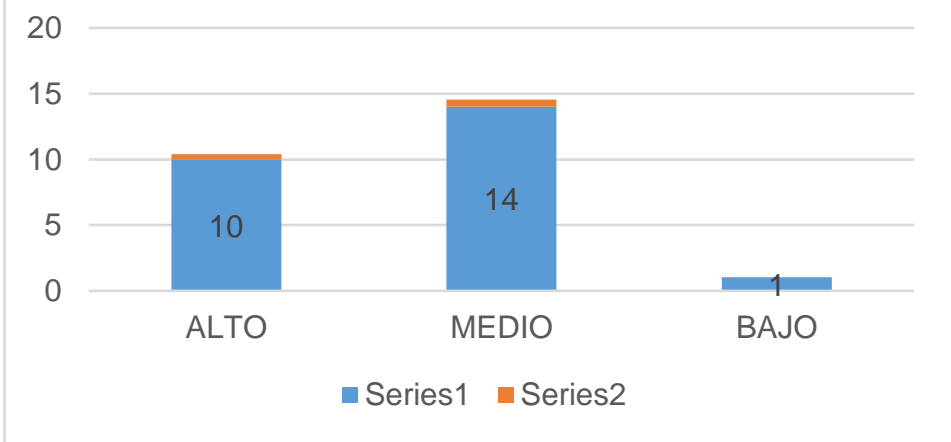

Gráfico 3

Fuente: Elaboración propia

Tabla 3. 


\begin{tabular}{cccc}
\hline \multicolumn{4}{c}{ ¿Cuál es el nivel de tecnología en la institución } \\
educativa donde labora?
\end{tabular}

Fuente: Elaboración propia

Rendimiento académico de estudiantes

Si: 24 docentes

No: 1 docente

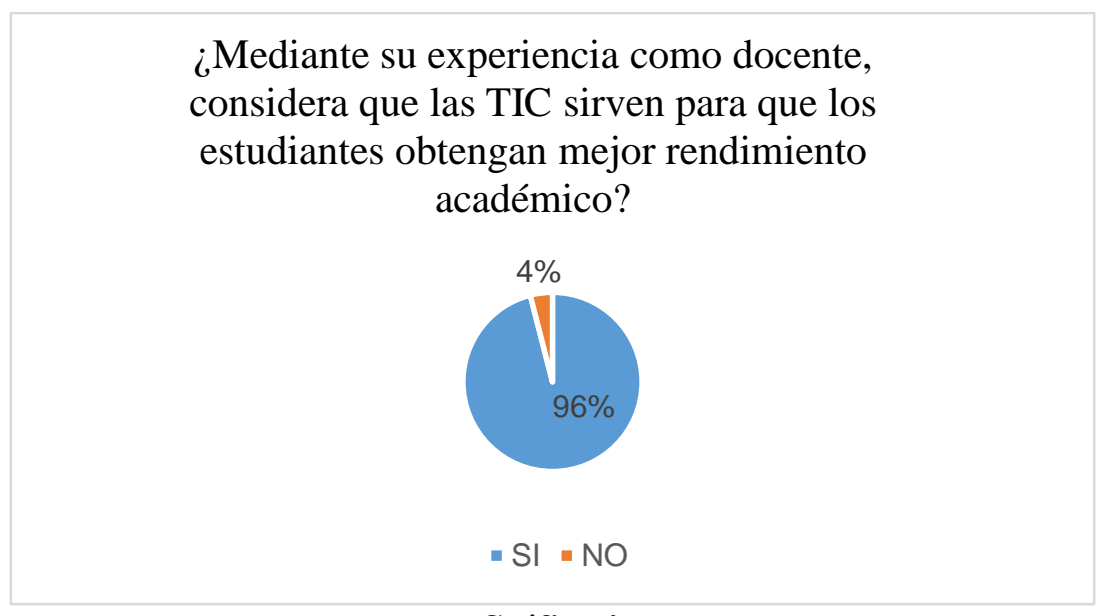

Gráfico 4.

Fuente: Elaboración propia

\section{Tabla 4.}

¿Mediante su experiencia como docente, considera que las TIC sirven para que los estudiantes obtengan mejor rendimiento académico?

\begin{tabular}{ccc}
\hline SI & NO & TOTAL \\
24 & 1 & 25 \\
$96 \%$ & $4 \%$ & $100 \%$ \\
\hline
\end{tabular}

Fuente: Elaboración propia

Los docentes respondieron, que no consideran que las TIC sean distractores dentro del proceso de enseñanza-aprendizaje en los estudiantes. Indican que el rendimiento depende de cada uno por medio de su responsabilidad e interés, más no por las TIC en general. 


\section{¿Considera que los docentes deben tener capacitación previa frente al uso de las TICS?}

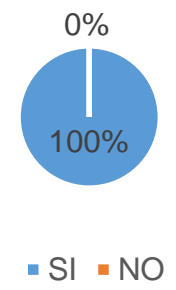

\section{Gráfico 5. Capacitación de docentes}

Fuente: Elaboración propia

\section{Tabla 5.}

¿Considera que los docentes deben tener capacitación previa frente al uso de las TICS?

\begin{tabular}{lll}
\hline SI & NO & TOTAL \\
25 & 0 & 25 \\
$100 \%$ & $0 \%$ & $100 \%$ \\
\hline
\end{tabular}

Fuente: Elaboración propia

Los docentes encuestados coincidieron en que todos deben recibir capacitaciones o charlas sobre el avance tecnológico para dar mejores explicaciones a los alumnos.

¿Qué tipo de materias enseña?

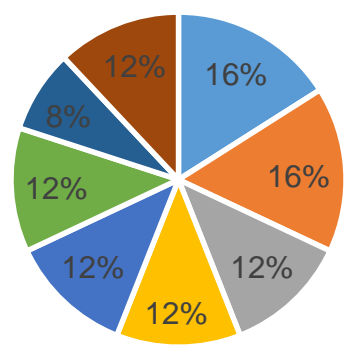

$$
\begin{array}{ll}
\text { - MATEMÁTICA } & \text { - LENGUA Y LITERATURA } \\
\text { - CIENCIAS SOCIALES } & \text { " CIENCIAS NATURALES } \\
\text { - COMPUTACIÓN } & \text { - IDIOMA EXTRANJERO } \\
\text { - CULTURA FÍSICA } & \text { - EDUCACIÓN ARTÍSTICA }
\end{array}
$$

Gráfico 6. Materias que imparte Fuente: Elaboración propia

\section{Tabla 6.}




\begin{tabular}{|c|c|c|c|c|c|c|c|c|}
\hline \multicolumn{9}{|c|}{ ¿Qué tipo de materias enseña? } \\
\hline 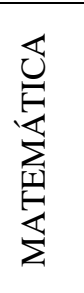 & 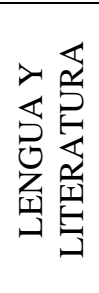 & 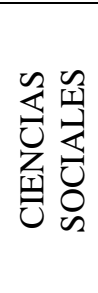 & 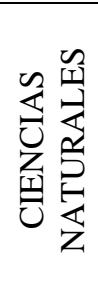 & 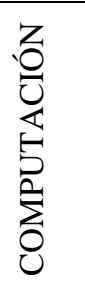 & 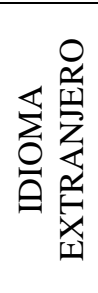 & 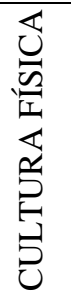 & 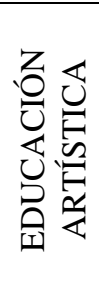 & $\begin{array}{l}\text { 它 } \\
0 \\
0\end{array}$ \\
\hline 4 & 4 & 3 & 3 & 3 & 3 & 2 & 3 & 25 \\
\hline $16 \%$ & $16 \%$ & $12 \%$ & $12 \%$ & $12 \%$ & $12 \%$ & $8 \%$ & $12 \%$ & $100 \%$ \\
\hline
\end{tabular}

Fuente: Elaboración propia

\section{Implementación tecnológica en la institución}

Si: 22 docentes

No: 3 docentes

\section{¿La unidad educativa cuenta con las \\ herramientas TICS necesarias para \\ complementar las clases dentro de las \\ aulas?}

$12 \%$



Gráfico 7.

Fuente: Elaboración propia

\section{Tabla 7.}

¿La unidad educativa cuenta con las herramientas TICS necesarias para complementar las clases dentro de las aulas?

\begin{tabular}{lll}
\hline SI & NO & TOTAL \\
22 & 3 & 25 \\
$88 \%$ & $12 \%$ & $100 \%$ \\
\hline
\end{tabular}

Fuente: Elaboración propia 


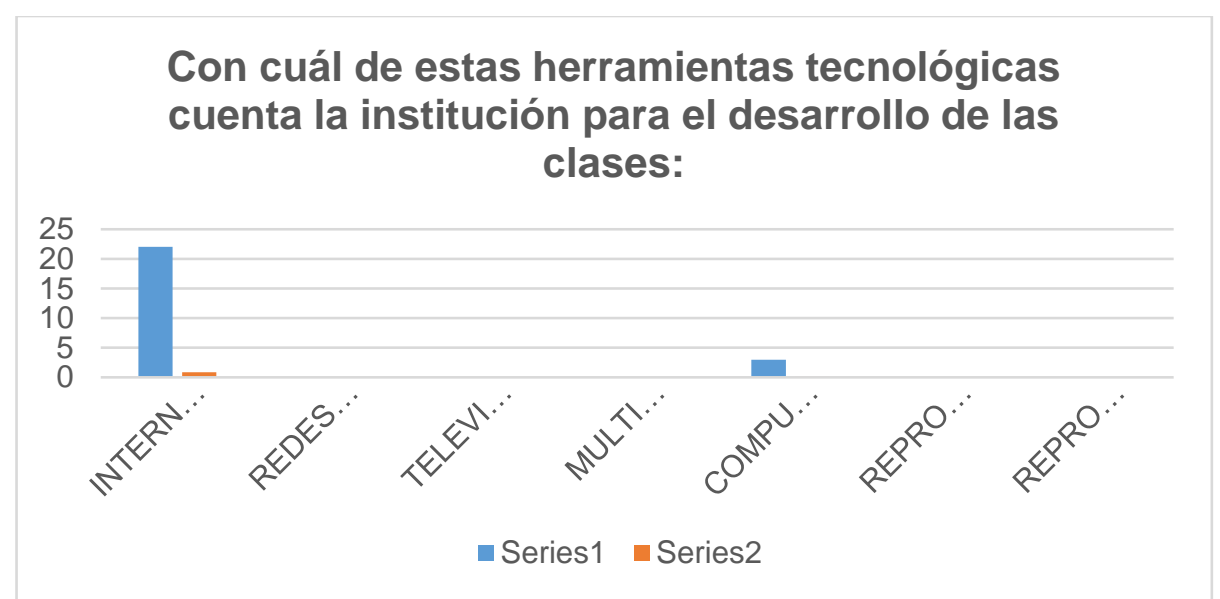

Gráfico 8. Herramientas tecnológicas que posee la institución Fuente: Elaboración propia

Tabla 8.

¿Con cuál de estas herramientas tecnológicas cuenta la institución para el desarrollo de las clases?

\begin{tabular}{|c|c|c|c|c|c|}
\hline 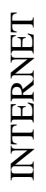 & 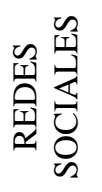 &  & 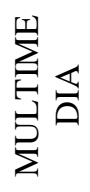 & 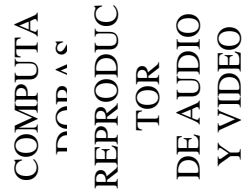 & 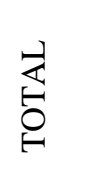 \\
\hline & $\begin{array}{c}22 \\
88 \%\end{array}$ & & & $\begin{array}{c}3 \\
12 \%\end{array}$ & $\begin{array}{c}25 \\
100 \%\end{array}$ \\
\hline
\end{tabular}

Fuente: Elaboración propia

Un total de 22 profesores coinciden en que la institución educativa tiene todas las herramientas tecnológicas necesarias para el desarrollo de las clases (internet, correo electrónico, redes sociales, computadoras, reproductores de audio, reproductores de video, televisión, multimedia), aunque también se destaca la opinión de 3 profesores, quienes manifestaron que la institución debe fortalecer este aspecto, ya que solamente se cuenta con herramientas básicas como computadoras, reproductores de audio y reproductores de video.

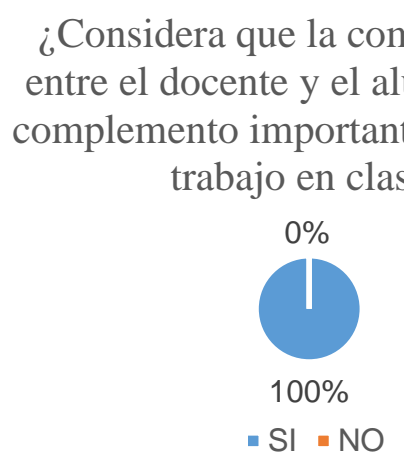

Gráfico 9. Complemento de las TIC en la comunicación entre el docente y estudiante Fuente: Elaboración propia 


\section{Tabla 9.}

\begin{tabular}{lll}
\hline $\begin{array}{l}\text { ¿En cuanto a las TIC en la educación, considera que la comunicación } \\
\text { entre el docente y el alumno es un complemento importante durante el } \\
\text { trabajo en clase? }\end{array}$ & \\
& & \\
\hline SI & NO & TOTAL \\
25 & 0 & 25 \\
$100 \%$ & $0 \%$ & $100 \%$ \\
\hline
\end{tabular}

Fuente: Elaboración propia

La buena comunicación que existe y se desarrolla entre docentes y estudiantes, es producto del trabajo realizado y que se basa en la interacción de la clase (docente-estudiante).

El aprendizaje es significativo y llega a los estudiantes de una manera más óptima. Todos coincidieron en la importancia de este punto.

\section{Conclusiones y recomendaciones}

Los docentes que se apoyan en el uso de las TIC, logran obtener una mejor enseñanza para sus estudiantes debido a que complementan y fortalecen su metodología al momento de impartir sus clases.

Es de vital importancia que se trabaje con las herramientas tecnológicas y exista una actualización constante en el uso de ellas. El desarrollo tecnológico en pleno siglo XXI, es un complemento vital dentro de la sociedad y por ende en la educación en todos sus niveles a nivel mundial.

Tanto las instituciones educativas públicas como privadas, deben ir fortaleciendo e incluir dentro de su plan académico de estudio, la utilización de las TIC para que los estudiantes empiecen a familiarizarse desde etapas tempranas con estas herramientas en busca de un mejor desempeño académico.

\section{Bibliografía}

Ángel, D.-B. (2013). Tic En El Trabajo Del Aula. Impacto En La Planeación Didáctica. Revista Iberoamericana De Educación Superior, 4(10), 3-21. Https://Doi.Org/10.1016/S20072872(13)71921-8

Asignatura, S., Asignatura, T. D. E., La, J., \& Social, C. (N.D.). Educación Y Desarrollo Comunicación Social Reforzar Y Ampliar El Conocimiento De Temas Claves Que Surgen En La Relación Entre Comunicación Y Educación, Analizando Cómo Desde Los Diferentes Medios De Comunicación Y A Través De Las Distintas Estrategias Se.

Belloch, C. (2012). Las Tecnologías De La Información Y Comunicación En El Aprendizaje. Unidad De Tecnología Educativa (Ute)- Departamento De Métodos De Investigación Y 
$\begin{array}{lllll}\text { Diagnostico } & \text { En } & \text { Educación., } & \text { 1-9. } & \text { Retrieved }\end{array}$ Http://Www.Uv.Es/Bellochc/Pedagogia/Eva1.Pdf

Carmona, K. O., Carmona, K. M. O., Armenta, J. A., Gastelú, C. A. T., \& García, E. M. M. (2016). Las Tic En Educación: Meta análisis Sobre Investigación Y Líneas Emergentes En México. Apertura, 8(2), 100-115. Https://Doi.Org/10.18381/Ap.V8n2.866

Castro, S. (2007). Las Tic En Los Procesos De Enseñanza Y Aprendizaje. Revista Científicas De América Latina, 13(23), 213-234. Https://Doi.Org/1315-883x

Cortés, J. A., Rigoberto, M., Uribe, M., \& Ibarra, I. G. (2013). Ámbitos Y Alcances De La Competencia Comunicativa En Educación. European Scientific Journal, 9(34), 18577881.

Ecuador, U. E. (S.F.). Recuperado El 2 De Diciembre De 2016, De Http://Providenciagye.Blogspot.Com/2012/04/La-Unidad-Educativa-De-LaProvidencia.Html

Gómez, L. M., \& Macedo, J. C. (2012). Importancia De Las Tic En La Educación. Investigación Educativa, 14(25), 209-224. $\quad$ Retrieved From Http://Revistasinvestigacion.Unmsm.Edu.Pe/Index.Php/Educa/Article/View/4776kaplunMario_Modelos-De-Educacic3b3n-Y-Comunicacic3b3n.Pdf. (N.D.). Of, I. (2012). Tic Educación : In, 10-12.

Gutiérrez, F., \& Mead, M. (1981). Educación Y Medios De Comunicación Social, 147-151.

Huergo, P. J. (1993). Los Medios Y Tecnologías En Educación, 1-21.

Mcluham, H. M. (2002). Comunicación Y Educación: El Proceso Didáctico Como Proceso De Comunicación. Artículos Para Comunicación, (1972), 1-7.

Moral Pérez, M. E. Del, Martínez, L. V., \& Neira Piñeiro, M. D. R. (2014). Oportunidades De Las Tic Para La Innovación Educativa En Las Escuelas Rurales De Asturias. Aula Abierta, 42(1), 61-67. Https://Doi.Org/10.1016/S0210-2773(14)70010-1

Muñoz-Miralles, R., Ortega-González, R., Batalla-Martínez, C., López-Morón, M. R., Manresa, J. M., \& Torán-Monserrat, P. (2014). Acceso Y Uso De Nuevas Tecnologías Entre Los J?Venes De Educación Secundaria, Implicaciones En Salud. Estudio Joitic. Atención Primaria, 46(2), 77-88. Https://Doi.Org/10.1016/J.Aprim.2013.06.001

Rúgeles, P. A., Mora, B., \& Metaute, P. M. (2015). El Rol Del Estudiante En Los Ambientes Educativos Mediados Por Las Tic. Revista Lasallista De Investigación, 12(2), 132-138. Retrieved From Http://Www.Redalyc.Org/Articulo.Oa?Id=69542291025

Unidad Educativa "De La Providencia". (S.F.). Recuperado El 2 De Diciembre De 2016, De Http://Delaprovidencia.Edu.Ec/Main.Aspx 
Vidal Puga, M. D. P. (2006). Investigación De Las Tic En La Educación. Relatec: Revista Latinoamericana De Tecnología Educativa, 5(2), 539-552. Retrieved From Http://Dialnet.Unirioja.Es/Servlet/Articulo?Codigo=2229253\&Info=Resumen\&Idioma=S pa 